\title{
Reduction of Patient Motion Artifacts in Digital Subtraction Angiography: Evaluation of a Fast and Fully Automatic Technique
}

\author{
Erik H. W. Meijering, Wiro J. Niessen, Jeannette Bakker, \\ Aart J. van der Molen, Gerard A. P. de Kort, Rob T. H. Lo, \\ Willem P. Th. M. Mali, Max A. Viergever
}

Radiology, vol. 219, no. 1, April 2001, pp. 288-293

\begin{abstract}
The performance of an automatic technique for reduction of patient motion artifacts in digital subtraction angiography (DSA) was evaluated. Four observers assessed the image quality of 104 cerebral DSA images, which were processed by both the automatic technique and manual pixel shifting. The automatic technique was found to result in better image quality and to be considerably less time consuming.
\end{abstract}

Keywords - Cerebral angiography, digital subtraction angiography, image artifacts, image processing, image registration, image quality.

\section{INTRODUCTION}

Patient motion artifacts are a major cause of image quality degradation in digital subtraction angiography (DSA). Although several techniques have been proposed over the past two decades to improve the acquisition of DSA images in relation to this problem [6], motion artifacts cannot be entirely avoided. Currently, the only post-processing techniques available on clinical DSA devices are manual remasking and pixel shifting, which allow for reduction of artifacts caused by uniform translational motion only [5,6]. Generally, however, patient movements have a more complex nature, which limits the effectiveness of these reduction techniques. This problem has been recognized by researchers in the field of image processing and has been the incentive to the development of a number of semi- or even full-automatic, nonlinear retrospective registration techniques [6]. However, apart from two exceptions $[4,8]$, clinical evaluations of these techniques have never been reported. Another major problem with these techniques is that they are generally too time consuming for use in clinical practice.

Recently, a new, fully automatic registration technique was developed, which is capable of aligning pairs of images nonlinearly within less than a second [7]. In this study we evaluated the effectiveness of this technique in reducing patient motion artifacts, by comparison with manual pixel shifting. The study was carried out on cerebral DSA images. 


\section{MATERIALS AND METHODS}

\section{Images and Equipment}

During a five-month period, 104 cerebral X-ray angiography runs from 21 patients (13 men and 8 women, age range 28-82 years) were archived digitally. From each run, we randomly selected one mask-contrast image pair, of which the corresponding DSA image had been printed on film by the radiologists at the time of the initial examination. All images had been acquired on an Integris V3000 C-arm imaging system (Philips Medical Systems, Best, the Netherlands), with a $20 \mathrm{~cm}$ or $25 \mathrm{~cm}$ image intensifier, a matrix size of either $512 \times 512$ pixels (31 images) or $1024 \times 1024$ pixels (73 images), and a grey-level resolution of 10 bits per pixel.

Post-processing operations as well as image quality assessments were carried out on an Octane ${ }^{\circledR}$ workstation (Silicon Graphics, De Meern, the Netherlands) with one $195 \mathrm{MHz}$ MIPS R10000 processor, 256MB main memory (instruction and data cache size both $32 \mathrm{~KB}$ ), and an IMPACTSR graphics board with 4MB texture memory. All images were displayed in a window of $700 \times 700$ pixels on a 19-inch monitor (Silicon Graphics, De Meern, the Netherlands), which had a resolution of $1280 \times 1024$ pixels (refresh rate $75 \mathrm{~Hz}$ ). By using this window, images were displayed with the same effective diameter $(\approx 11.5$ inch $)$ as they are usually displayed on the 15-inch progressive display monitor of the Integris V3000. The contrast and brightness settings of the window were fixed during the evaluation.

\section{Manual and Automatic Registration}

Manual correction for motion artifacts in the DSA images corresponding to the 104 maskcontrast pairs was obtained by using a special pixel-shifting tool that could be executed on the Octane ${ }^{\circledR}$ workstation and that exactly mimiced the pixel-shifting facility on the viewing console of the Integris V3000 used in daily practice. Automatic correction was obtained by using the algorithm by Meijering et al. [7], briefly described below (see also Figure 1). First, the algorithm applies edge detection to the mask image in order to extract regions that have a high potential for showing artifacts. Next, control points for the warping operation are automatically selected at local maxima of the gradient magnitude, while constraining the minimum and maximum distance between these points. The local displacements of image structures at the control points are then computed by means of a template matching procedure based on the energy similarity measure [2], followed by detection and correction of inconsistent displacement vectors by comparison with neighboring vectors. Finally, the mask image is warped according to the displacement vector field resulting from linear interpolation of the local displacements at the control points. This is done very efficiently by using a triangulation of the control points in combination with hardware accelerated texture-mapping. For the present study, all parameters of the algorithm were fixed to the proposed values [7].

\section{Method of Evaluation}

Four observers (three radiologists and a resident) participated in the evaluation, which consisted of two parts. In the first part, manual registrations of the 104 mask-contrast image pairs were carried out separately and independently by the four observers, using the pixel-shifting tool. Since optimal manual registration of an image pair is task dependent, the observers were provided with the clinical indication for acquisition of the images, which was either a cerebral aneurysm (41 images; 7 patients), a stenosis in the carotid 

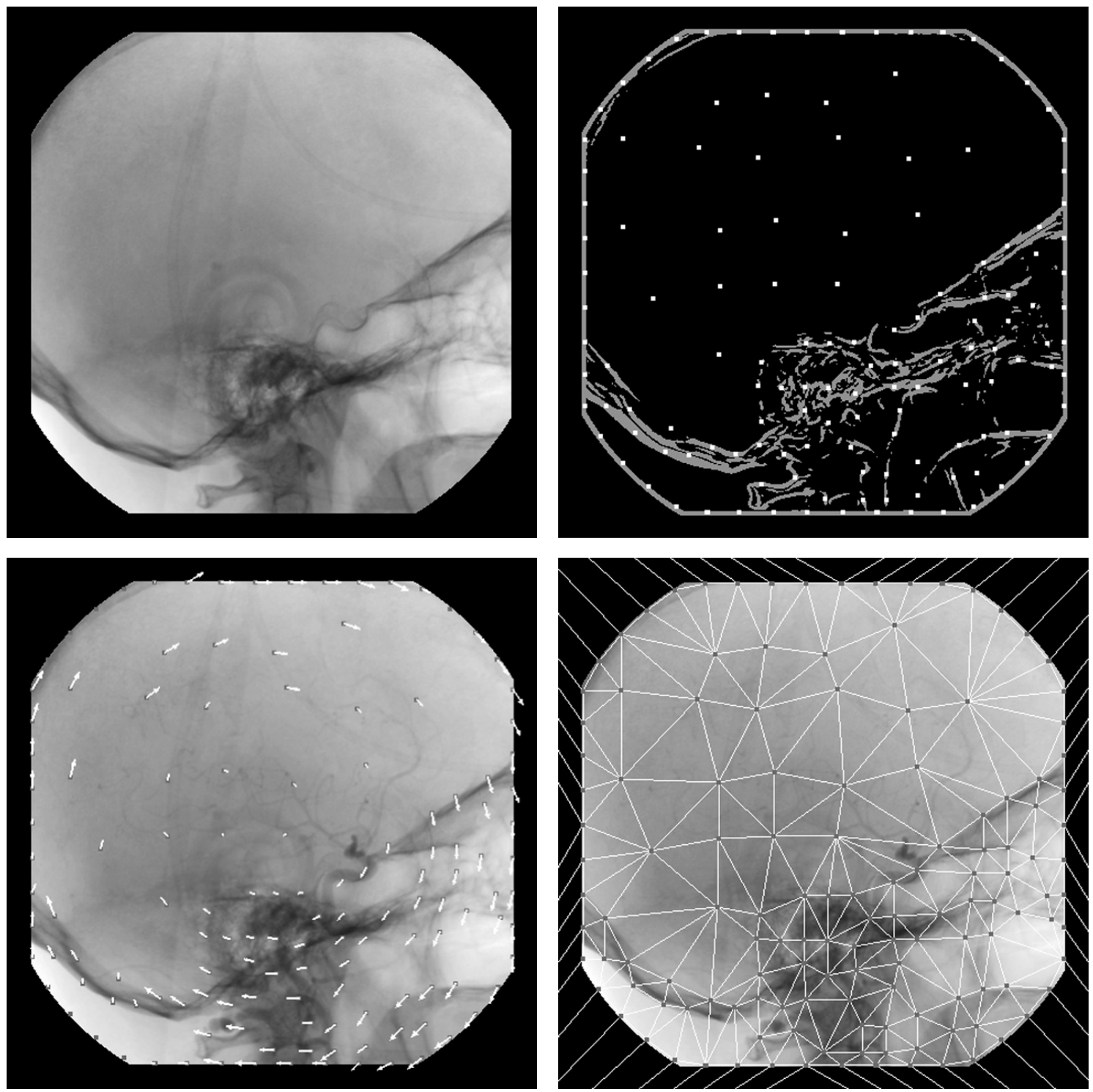

Figure 1. Example of the several stages in the automatic registration algorithm evaluated in this study. Top-left: mask image of a lateral cerebral DSA image. Top-right: output of the edge detection algorithm (grey regions) and control-point selection mechanism (white dots). Bottom-left: live image overlayed with the automatically computed local displacement vectors, indicating the correspondence with the mask image. From the vector field it is clear that in this example the patient's movement was of a rotational nature. Bottomright: triangulation of the set of control points used for interpolation of the displacement vectors and final warping of the mask image.

arteries (34 images; 8 patients), a tumor (20 images; 4 patients), or vasculitis (9 images; 2 patients). However, the images were presented in random order. The final horizontal and vertical mask shifts for all mask-contrast pairs as indicated by each of the observers were stored automatically by the computer, together with the time it took for each observer to carry out the manual registration of each pair. The resulting manually corrected DSA images were also stored. The DSA images resulting from automatic registration of all mask-contrast pairs were computed and stored separately. 
The second part of the study concerned the comparison of the quality of the automatically and manually corrected DSA images. To this end, the following three DSA image pairs were formed for each of the 104 original DSA images: (i) the automatically corrected DSA image and the original DSA image, (ii) the manually corrected DSA image and the original DSA image, and (iii) the automatically corrected DSA image and the manually corrected DSA image. This resulted in a total of 312 DSA image pairs, which were presented to the observers. Although the original and automatically corrected DSA images were the same for all four observers, each of the observers was confronted with his or her own manual corrections resulting from the first part. For each of the DSA image pairs, the differences between the two images (denoted "Image A" and "Image B") could be assessed by alternating the image that was displayed.

The observers were given the clinical information of all images and were asked to rate the relative quality of the two images by choosing one of the following: (AB) Image A and Image $\mathrm{B}$ are similar (i.e., the number of artifacts and the magnitude of the artifacts is the same in the diagnostically relevant parts or in the entire images), $(\mathrm{A}+)$ Image $\mathrm{A}$ is better than, or $(\mathrm{A}++)$ much better than Image $\mathrm{B}$ (i.e., the number of artifacts or the magnitude of the artifacts in Image A is smaller, or much smaller than in Image B, in the diagnostically relevant parts or in the entire image), $(\mathrm{B}+)$ Image $\mathrm{B}$ is better than, or $(\mathrm{B}++)$ much better than Image $\mathrm{A}$ (i.e., the number of artifacts or the magnitude of the artifacts in Image B is smaller, or much smaller than in Image A, in the diagnostically relevant parts or in the entire image).

Similar to the first part, the second part of the study was carried out separately and independently by the four observers. However, prior to this part, there was a meeting between the observers in order to obtain consensus regarding the rating of relative image quality. For this consensus meeting, 10 sample cerebral DSA image pairs were used, which were not included in the actual study.

To avoid bias in the ratings, the images were presented to the observers in a completely randomized and blinded fashion; not only were the 312 DSA image pairs randomized, but also the order of the images within each pair was randomized, and the observers were ignorant of the type of correction (no, manual, or automatic correction) that was applied to the images. Furthermore, to reduce the possibility of observers recognizing their own manual corrections, the time period between the first and the second part of the study was at least three weeks for each of the observers.

\section{Statistical Analyses}

Inter-observer agreement for the image quality ratings resulting from the second part of the study was assessed by using a kappa $(\kappa)$ test. In order to take account of the degree of disagreement, we used the weighted kappa $\left(\kappa_{\mathrm{w}}\right)$ test, the weights for discrepancies of 0 , $1,2,3$, and 4 categories in the ratings being $1,0.75,0.5,0.25$, and 0 , respectively $[1,3]$. Six $\kappa_{\mathrm{w}}$ values were computed, based on a comparison of the ratings of two observers at a time. A $\kappa_{\mathrm{w}}$ value of 1.0 indicates that the agreement is perfect and a value of 0.0 that it is not different from chance agreement. For the interpretation of $\kappa_{\mathrm{w}}$ values in between these extremes, we used the Landis-Koch guidelines [1].

The ratings resulting from the second part allowed us to make both implicit and explicit comparisons of the effectiveness of the automatic and the manual registration technique in reducing motion artifacts. For this purpose, the ratings of the 312 DSA image pairs were divided into three groups: (i) ratings expressing the quality of automatically corrected DSA images relative to corresponding original (uncorrected) DSA images (or vice versa), (ii) 


\begin{tabular}{lcccccc}
\hline \hline & \multicolumn{5}{c}{ Original rating } \\
Relative image quality & $\mathrm{A}++$ & $\mathrm{A}+$ & $\mathrm{AB}$ & $\mathrm{B}+$ & $\mathrm{B}++$ \\
\hline Image A compared to Image B & ++ & + & 0 & - & -- \\
Image B compared to Image A & -- & - & 0 & + & ++ \\
\hline \hline
\end{tabular}

Table 1. Rules for conversion of the original ratings resulting from the second part of the study, necessary in order to be able to express the quality of any one of the DSA images in a given pair in terms of the other.

ratings expressing the quality of manually corrected DSA images relative to corresponding original (uncorrected) DSA images (or vice versa), and (iii) ratings expressing the quality of automatically corrected DSA images relative to corresponding manually corrected DSA images (or vice versa). Since the images of each pair were presented in random order, the original ratings were converted by using the rules presented in Table 1 in order to be able to express the quality of any of the images in a given pair in terms of the other. Implicit comparison of the performance of the automatic and manual registration technique was then obtained by constructing a frequency table of the converted ratings from groups (i) and (ii). Explicit comparison was obtained by analyzing the ratings from group (iii). These comparisons were carried out separately for the results of each observer. A comparison based on the average frequencies was also carried out.

The statistical significance of the possible improvement of the automatic registration technique over manual pixel shifting was assessed by using a Chi-squared $\left(\chi^{2}\right)$ test $[1,3]$ applied to the frequency tables containing the ratings from groups (i) and (ii). Since one of the variables in these tables (viz., relative image quality) represents ordered categories, we did not use the ordinary $\chi^{2}$ test, but the more powerful $\chi^{2}$ test for linear trend $[1,3]$. For this test, we used uniform spacing of the categories. The null hypothesis was that the automatic and manual registration technique would be equally effective in reducing motion artifacts. A probability of $p<0.05$ for this hypothesis was chosen to indicate a statistically significant difference between the two techniques.

\section{RESULTS}

In the first part of the study, the observers found that most of the 104 original cerebral DSA images could be improved to some extent by manual correction, since on average $88 \%$ of the mask shift parameters differed from zero. The maximum shift recorded in either direction was 8.0 pixels, while the average length of the shift vectors of all four observers was 1.2 pixels. From this it may be concluded that, although in some cases patient motion was quite severe, in most cases the motion artifacts were due to relatively small displacements only. The timing information stored along with the shift parameters revealed that manual correction required on average about $12 \mathrm{sec}$. (median: $7 \mathrm{sec}$.) per DSA image. In contrast, the automatic registration algorithm required on average only about 1 sec. (median: 1 sec.) per DSA image.

The $\kappa_{\mathrm{w}}$ values computed from the ratings in the second part ranged from 0.60 to 0.71 . According to the Landis-Koch guidelines, this indicates substantial agreement. Therefore, we restrict ourselves to presenting averages. The average frequencies of the ratings from groups (i) and (ii), as described in Section, are presented in Table 2. From the implicit comparison based on the results in this table it follows that, overall, the automatic regis- 


\begin{tabular}{lrrrrr}
\hline \hline & \multicolumn{5}{c}{ Relative image quality } \\
Comparison & -- & - & 0 & + & ++ \\
\hline Manually corrected versus original & $0 \%$ & $4 \%$ & $25 \%$ & $62 \%$ & $9 \%$ \\
Automatically corrected versus original & $0 \%$ & $0 \%$ & $15 \%$ & $69 \%$ & $16 \%$ \\
\hline \hline
\end{tabular}

Table 2. Average frequencies of the ratings resulting from the comparison of corrected DSA images and their corresponding original (uncorrected) DSA images. Manual corrections were obtained by using the pixel shifting technique, and automatic corrections by using the technique described by Meijering et al. [7].

\begin{tabular}{lcccccc}
\hline \hline & \multicolumn{5}{c}{ Relative image quality } \\
Comparison & - & - & 0 & + & ++ \\
\hline Automatically versus manually corrected & $0 \%$ & $5 \%$ & $44 \%$ & $48 \%$ & $3 \%$ \\
\hline \hline
\end{tabular}

Table 3. Average frequencies of the ratings resulting from the explicit comparison of automatically corrected DSA images and their corresponding manually corrected DSA images. Manual corrections were obtained by using the pixel shifting technique, and automatic corrections by using the technique described by Meijering et al. [7].

tration technique resulted in better image quality than manual pixel shifting. In order to be able to apply the $\chi^{2}$ test for trend, the frequencies in the columns "- -" and "-" had to be combined, since this test does not allow rows or columns to be entirely filled with zeroes. The $\chi^{2}$ test for trend applied to the modified frequency table showed that the probability for the null hypothesis of equal effectiveness to be true is $p<0.05$, from which it can be concluded that the automatic correction technique is statistically significantly better than manual pixel shifting in reducing motion artifacts. We note that the same conclusion was found with this test applied to the results of the observers separately. The average frequencies of the ratings from group (iii), representing the results of the explicit comparison of the quality of automatically and manually corrected images, are presented in Table 3. These results support the conclusion drawn from the implicit comparison.

Two examples of cases in which the automatic registration technique was found to be superior to manual pixel shifting are given in Figures 2 and 3, where the artifacts in the original DSA images are located primarily in the lower part of the image, around the main vessels. Although the artifacts could be removed to some extent by manual pixel shifting, it was not possible to completely remove them using this technique. In the case of Figure 2, pixel shifting even resulted in a deterioration of artifacts in the lower right part of the image. Application of the automatic registration technique, on the other hand, resulted in overall correction and improved vessel visibility.

\section{DISCUSSION}

Of the 104 DSA images included in this study, 38 (37\%) had been manually corrected by means of pixel shifting before being printed on film and stored in the archive of our hospital after initial examination. From the fact that, in the first part of the study, the observers found that no less than $92(88 \%)$ of the images could be improved to some extent 

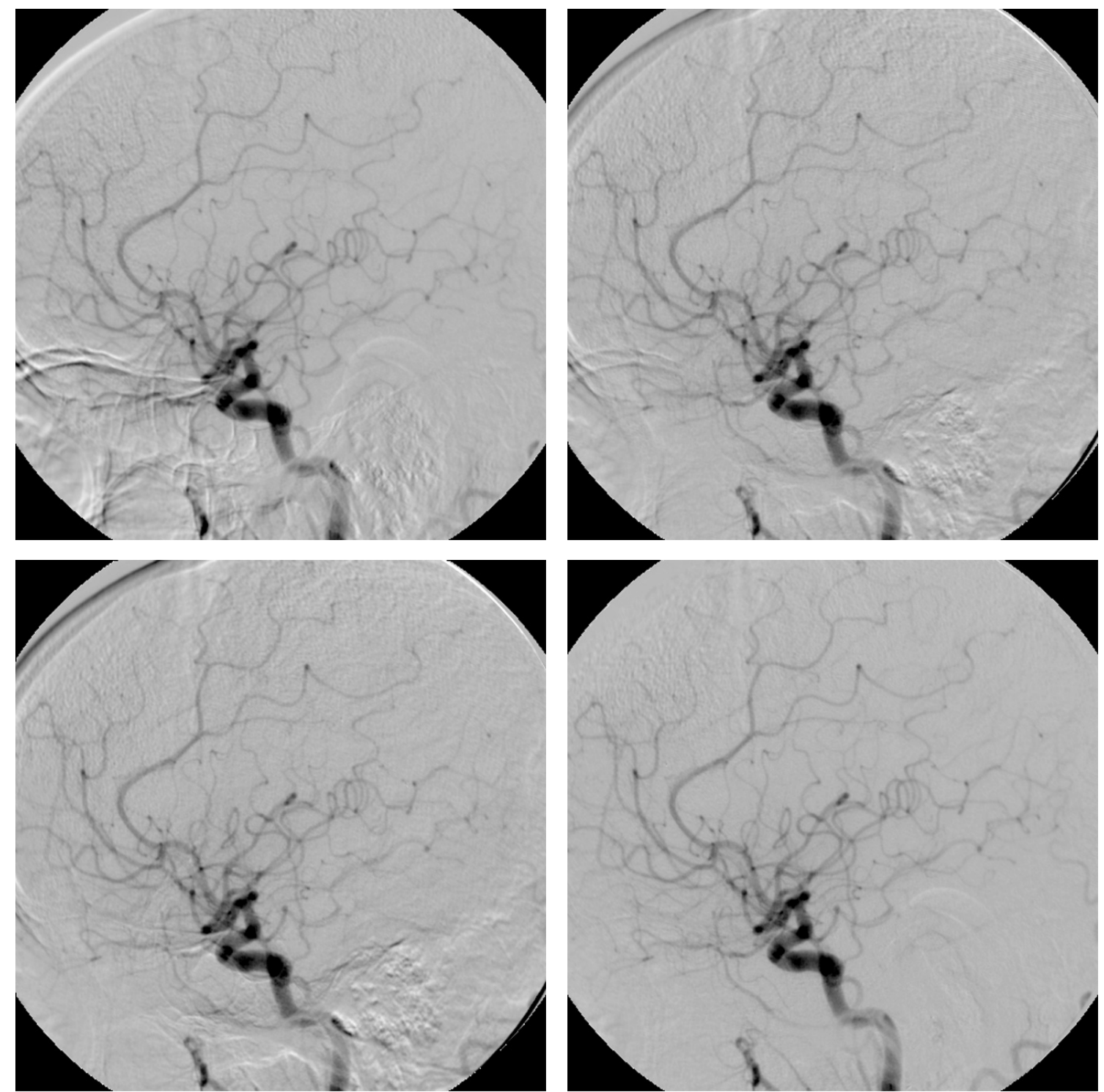

Figure 2. First example of a case in which the automatic registration technique was found to be superior compared to manual pixel shifting. Top-left: original lateral cerebral DSA image. Top-right and bottom-left: resulting DSA image after manual registration by means of pixel shifting, for two of the four observers. Due to the rotational nature of the patient's movement, it was not possible to obtain an overall optimal correction of motion artifacts by means of this technique. From these two images it is clear that a reduction of artifacts in one part of the image (in this example the lower left part) may result in a deterioration of artifacts elsewhere (in this example the lower right part). bottom-right: DSA image resulting from application of the automatic registration technique.

by this technique, we conclude that in practice more images contain motion artifacts than are usually corrected. Manual correction of all images by means of pixel shifting is a labor intensive operation: the results of our study indicated that, on average, 12 sec. per DSA image are required to apply this technique optimally. Apart from resulting in better overall image quality than manual pixel shifting, the automatic technique is considerably faster. On average, it requires only 1 sec. per DSA image. Moreover, the technique does not require any effort from the radiologist. 


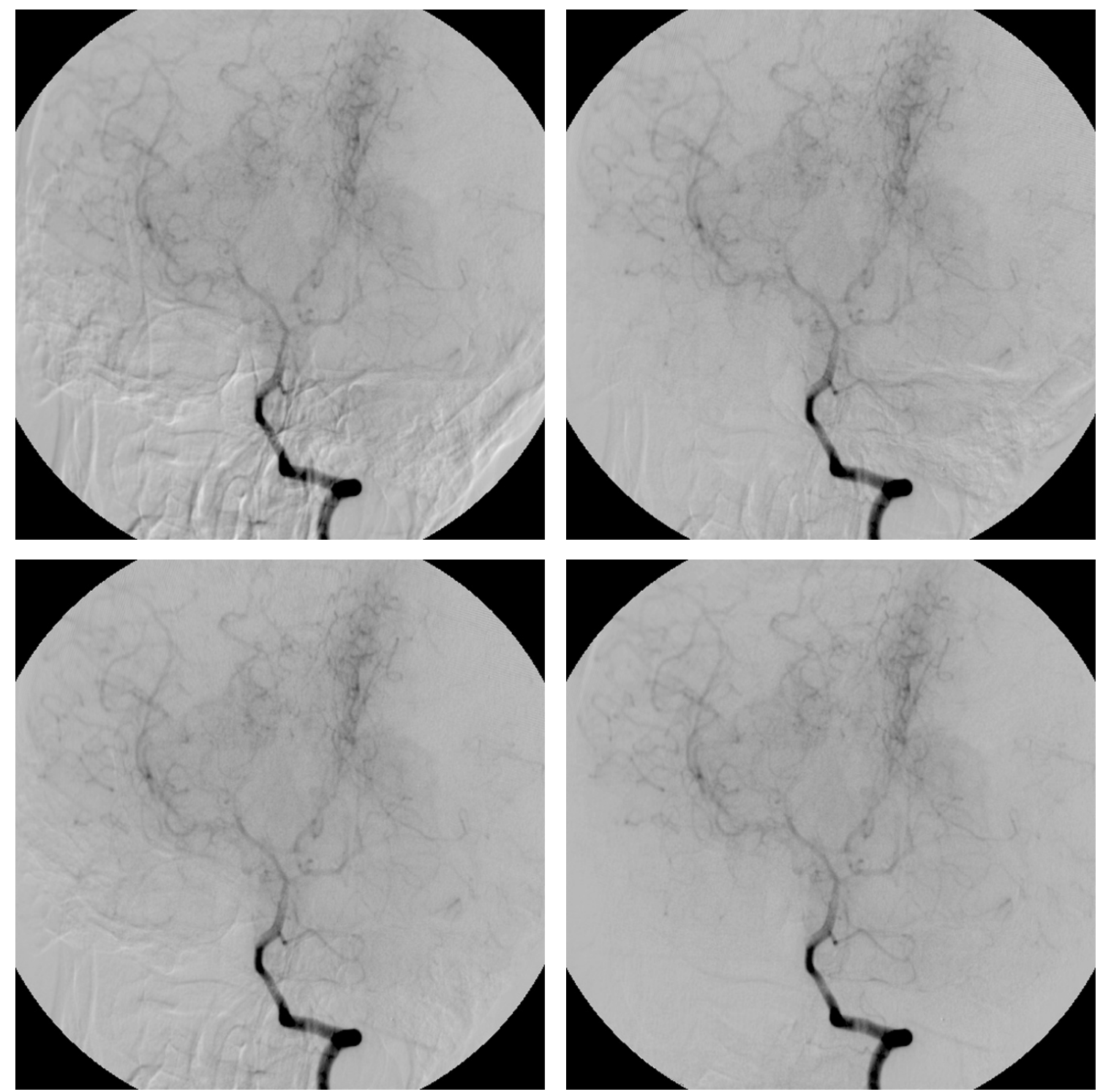

Figure 3. Second example of a case in which the automatic registration technique was found to be superior to pixel shifting. Top-left: original tilted oblique frontal cerebral DSA image. Top-right and bottom-left: resulting DSA image after manual registration by means of pixel shifting, for two of the four observers. Similar to the previous example, the patient's movement as projected in the imaging plane was more complex than uniform translation. Although the artifacts were reduced subtantially (see the lower part of the image), it was not possible to obtain an overall optimal correction of motion artifacts by means of this technique. Bottom-right: DSA image after application of the automatic registration technique, resulting in improved visibility of the main vessel.

We note that although the $\kappa_{\mathrm{w}}$ test indicated substantial agreement between the observers, there was some spread in the individual $\kappa_{\mathrm{w}}$ values. This may be due to the fact that in the second part of the study, the observers were confronted with their own manual corrections, which were sometimes different for the different observers. Furthermore, although the $\chi^{2}$ test for trend - applied to either the average results or the results of the individual observers - indicated a statistically significant difference $(p<0.05)$ in 
image quality after application of the automatic correction technique and manual pixel shifting, the outcome of an ordinary $\chi^{2}$ test would have been somewhat less persuasive: for two of the observers, this test would have given $p<0.1$ for the null hypothesis to be true. However, the $\chi^{2}$ test for trend is more appropriate, since we are dealing with ordered categories.

As clearly illustrated by the examples in Figures 2 and 3, manual pixel shifting often results in improved image quality in and near the diagnostically relevant parts of an image, but may sometimes result in deterioration of artifacts in other parts. This is a direct consequence of the fact that with this technique, patient motion as projected in the imaging plane is assumed to be uniform translational. One may argue that this is not really a problem in practice as long as artifacts can be reduced in the diagnostically relevant parts of the image, and that it is therefore sufficient to use manual pixel shifting rather than a more sophisticated automatic correction technique. However, even if there would be no difference in performance from the point of view of image quality, it would still be advantageous to use the automatic technique evaluated in this paper, since it is considerably less time consuming.

The fact that the automatic registration technique performed statistically significantly better than manual pixel shifting does not imply that in practice the former technique will always be better than the latter. The average results from the explicit comparison of the two techniques (Table 3 ) indicated that in $5 \%$ of all cases, the corrected DSA image resulting from manual pixel shifting was found to be better than the corresponding automatically corrected DSA image. We observed that in these cases the automatic technique did not introduce new artifacts, but it was unable to reduce some of the artifacts at the borders of the images. This may be caused by the lack of image content in those regions, which reduces the possibilities for any template matching procedure to find the correct local displacement vectors.

In most reports on reduction of motion artifacts in DSA images, the evaluation of newly developed techniques involved only one or at most a few clinical DSA images, or phantoms, and the quality of the resulting corrected images was assessed by the same persons that developed the algorithm. To the best of our knowledge, the only more elaborate and objective evaluation studies are the ones by Takahashi et al. [8] and Hayashi et al. [4]. In the former study, three techniques were evaluated: manual remasking, manual pixel shifting, and an automatic registration technique. It was concluded that remasking was most effective. It was also found that, after having applied remasking, remaining artifacts were reduced equally well by manual pixel shifting and their automatic registration technique. In the study of Hayashi et al. [4], the authors compared the performance of two techniques: manual pixel shifting and an automatic registration technique developed by some of the co-authors of that study. In 14 out of 16 cerebral DSA image series, the images resulting from the automatic registration technique were found to have better quality. In the other two cases, the techniques were found to perform comparably.

Due to the lack of detailed information provided by the authors of these papers, it is difficult to explicitly compare their findings to ours. We note, however, that the automatic registration technique evaluated in our study is based on approaches which have been shown to yield faster and more accurate registrations compared to their techniques (see Meijering et al. [6,7] for more technical details). Hayashi et al. [4] reported that their algorithm required about eight minutes of computation time. In contrast, the algorithm evaluated in our study requires on average only about 1 sec. per DSA image, which certainly makes it more suitable for use in clinical practice. Furthermore, owing to the use of better similarity measures in the template matching procedure, the images processed 
by our algorithm are either comparably to, better than, or even much better than those resulting from manual pixel shifting in $95 \%$ of all cases.

Finally, we mention the fact that our study involved only images that were already considered clinically useful. Frequently it occurs that, during acquisition, the patient's movements are too severe to result in diagnostically useful DSA images, even when using pixel shifting afterwards, and in such cases the run is repeated. In some cases, the automatic registration technique might help avoid a second DSA run. On-line availability of the automatically corrected DSA images would offer the radiologist the possibility to check directly whether a new run must be acquired, thereby avoiding the need to go back to the console to check it manually by means of pixel shifting. We also note that in our study, we were only interested in overall image quality improvement, without relation to specific diagnostic tasks, such as the grading of stenoses or the detection of small aneurysms. It may be that the automatic registration technique also implies an improvement in that respect compared to manual pixel shifting. Confirmation of these claims is the goal of future studies.

\section{ACKNOWLEDGMENTS}

The research described in this paper was carried out at the University Medical Center Utrecht (UMCU), the Netherlands, and was financially supported by the Netherlands Ministry of Economic Affairs. The authors are grateful to Philips Medical Systems (Best, the Netherlands) for making available their clinical Octane ${ }^{\circledR}$ workstation (Silicon Graphics, De Meern, the Netherlands) on which the evaluation was carried out. Gerard van Hoorn, Tineke Kievit, Wilma Pauw, Koen Vincken, Theo van Walsum, Remko van der Weide, and Onno Wink (all with the UMCU) are acknowledged for their assistance in the start-up phase of this study.

\section{REFERENCES}

[1] D. G. Altman, Practical Statistics for Medical Research, Chapman \& Hall, London, UK, 1991.

[2] T. M. Buzug \& J. Weese, "Image Registration for DSA Quality Enhancement", Computerized Medical Imaging and Graphics, vol. 22, no. 2, 1998, pp. 103-113.

[3] J. L. Fleiss, Statistical Methods for Rates and Proportions, 2nd ed., Wiley Series in Probability and Mathematical Statistics, Wiley, New York, USA, 1981.

[4] N. Hayashi, T. Sakai, M. Kitagawa, R. Inagaki, N. Sadato, Y. Ishii, Y. Nishimoto, M. Tanaka, T. Fukushima, H. Komuro, H. Ogura, H. Kobayashi, T. Kubota, "Nonlinear Geometric Warping of the Mask Image: A New Method for Reducing Misregistration Artifacts in Digital Subtraction Angiography", CardioVascular and Interventional Radiology, vol. 21, no. 2, 1998, pp. 138-141.

[5] D. C. Levin, R. M. Shapiro, L. M. Boxt, L. Dunham, D. P. Harrington, D. L. Ergun, "Digital Subtraction Angiography: Principles and Pitfalls of Image Enhancement Techniques", American Journal of Roentgenology, vol. 143, no. 3, 1984, pp. 447-454.

[6] E. H. W. Meijering, W. J. Niessen, M. A. Viergever, "Retrospective Motion Correction in Digital Subtraction Angiography: A Review", IEEE Transactions on Medical Imaging, vol. 18, no. 1, 1999, pp. $2-21$.

[7] E. H. W. Meijering, K. J. Zuiderveld, M. A. Viergever, "Image Registration for Digital Subtraction Angiography", International Journal of Computer Vision, vol. 31, no. 2/3, 1999, pp. 227-246.

[8] M. Takahashi, J. Shinzato, Y. Korogi, K. Fukui, S. Ueno, I. Horiba, N. Suzumura, "Automatic Reregistration for Correction of Localized Misregistration Artifacts in Digital Subtraction Angiography of the Head and Neck", Acta Radiologica (Supplementum), vol. 369, 1986, pp. 281-284. 